\title{
Inward Rectifier Current Downregulation Promotes Spontaneous Calcium Release in a Novel Model of Rat Ventricular Electrophysiology
}

\author{
Harley J Stevenson-Cocks, Michael A Colman, Ed White, Alan P Benson
}

School of Biomedical Sciences, University of Leeds, Leeds, United Kingdom

\begin{abstract}
Aberrant intracellular calcium handling, as observed in diseases such as heart failure, promotes lethal ventricular arrhythmias and sudden cardiac death. Recent data from our laboratory suggests that reduced expression of the inward rectifier current in failing rat myocytes increases spontaneous calcium release, however existing computational models are unable to reproduce the underlying stochastic calcium cycling dynamics and so we have been unable to use simulation approaches to explore the cause of this pro-arrhythmic behaviour.

Here, we develop a novel model of rat ventricular electrophysiology that reproduces normal spatio-temporal calcium dynamics. Simulations implementing a similar reduction in inward rectifier current to that observed experimentally show that spontaneous calcium release is promoted by action potential prolongation and sarcoplasmic reticulum loading in the presence of a depolarised resting membrane potential. Combined, these effects can result in triggered activity.

The model therefore provides insight into arrhythmogenic mechanisms in failing ventricular myocytes and can be utilised to further explore pro-arrhythmic behaviour caused by abnormal calcium handling.
\end{abstract}

\section{Introduction}

Heart failure (HF) is characterised by an inability of the heart to supply blood to the body, as the ventricles are unable to sufficiently contract or relax in a synchronous manner. Many HF patients die suddenly, largely from ventricular arrhythmias [1] which result in the rapid and terminal reduction of organ perfusion pressure. With the growing prevalance of cardiovascular disease globally, the development of improved preventative and therapeutic strategies is of paramount importance. Thus, greater understanding of the complex underlying processes is necessary to reduce the mortality, morbidity and economic burden of HF.

Aberrant calcium $\left(\mathrm{Ca}^{2+}\right)$ handling is known to contribute to both the mechanical and electrical dysfunction seen in HF, however dissecting the underlying mechanisms presents a major research challenge as they occur from the meso- to the macroscopic scale and across varying timescales. Accordingly, methodologies beyond traditional experimental ones are increasingly being employed to investigate the pathophysiological processes that result in these disease states. Sophisticated computational models of cardiac electrophysiology have been developed over the past $50+$ years that incorporate a myriad of processes, in one to three dimensions, as well as their associated heterogeneities [2].

Yet, despite the common use of the rat as an animal model of cardiovascular disease, existing computational models of $\mathrm{Ca}^{2+}$ handling in rat are unable to recreate many experimental observations and so are incapable of offering mechanistic insight. Our laboratory has recently shown that a reduction in inward rectifier channel $\left(I_{\mathrm{K} 1}\right)$ expression promotes spontaneous $\mathrm{Ca}^{2+}$ release in failing rat ventricular myocytes [3] (Figure 1), but current rat myocyte computational models do not capture the stochasticity of $\mathrm{Ca}^{2+}$ cycling and so it has not been possible to explore this observation further. Furthermore, existing rat electrophysiology models are unable to reproduce action potentials at the fast physiological rates observed experimentally in rat $(6-8 \mathrm{~Hz}$, e.g. in $[4,5])$.

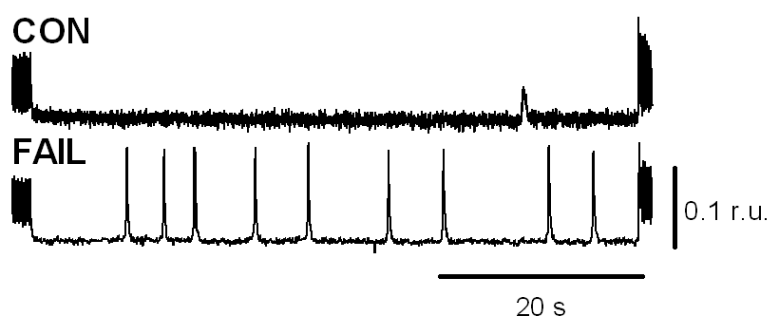

Figure 1. Intracellular $\mathrm{Ca}^{2+}$ in control $v s$ failing rat ventricular myocytes. Reduced $I_{\mathrm{K} 1}$ channel expression was found in HF myocytes, which exhibited frequent and substantial spontaneous $\mathrm{Ca}^{2+}$ release.

In this study, we develop a novel model of the rat ventricular myocyte which recreates normal electrophysiology and spatio-temporal $\mathrm{Ca}^{2+}$ dynamics at physiological 


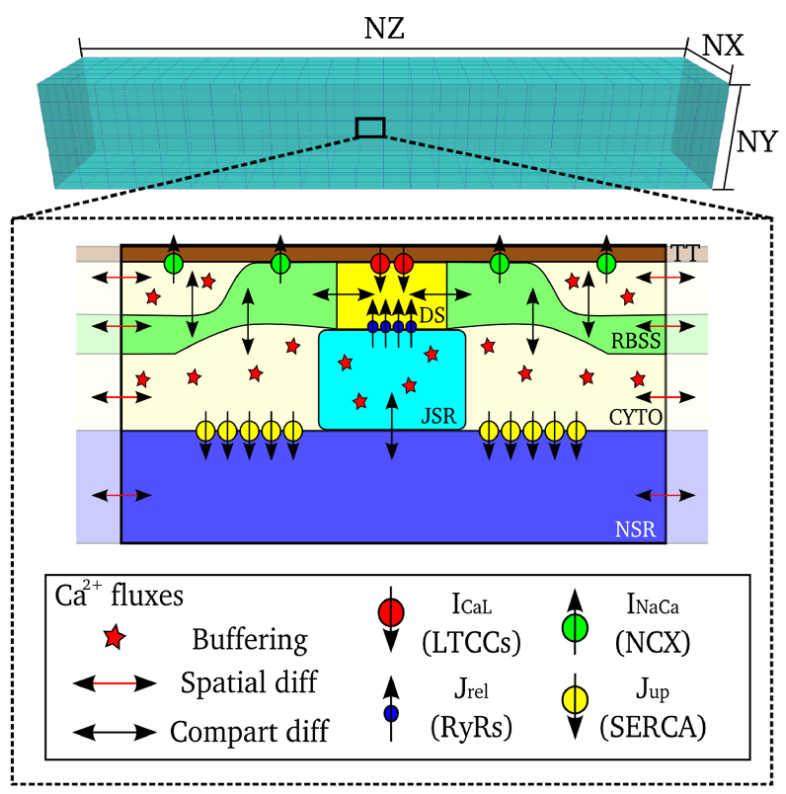

Figure 2. Structure of the spatio-temporal $\mathrm{Ca}^{2+}$ handling model. The model accounts for a variable number of calcium release units (CRUs, typically 20,000) each containing multiple compartments (DS, dyadic cleft space into which $\mathrm{Ca}^{2+}$-induced $\mathrm{Ca}^{2+}$ release occurs; SS, sub-space; CYTO, bulk cytosolic space; JSR, junctional sarcoplasmic reticulum which interacts with the DS; NSR, network SR). Diffusion occurs between the SS, CYTO and NSR spaces of neighbouring compartments but is restricted within each compartment for the JSR and DS. The membrane currents $I_{\mathrm{CaL}}$ and $I_{\mathrm{NaCa}}$ are present on the t-tubule (TT) membrane, and the fluxes $J_{\text {up }}$ and $J_{\text {rel }}$ control $\mathrm{Ca}^{2+}$ uptake and release, respectively, from the SR.

heart rates for the rat. We use this newly-developed model to investigate the effects of $I_{\mathrm{K} 1}$ downregulation on proarrhythmic $\mathrm{Ca}^{2+}$ handling behaviour in a simulation study.

\section{Methods}

A novel computational model was constructed by combining a recent model of rat ventricular electrophysiology [6] with a model of stochastic spatio-temporal $\mathrm{Ca}^{2+} \mathrm{cy-}$ cling developed in our laboratory [7] (Figure 2), which was then parameterised and validated against experimental data collected at various pacing frequencies. This model served as a control, from which a HF variant was generated by reducing the $I_{\mathrm{K} 1}$ current conductance parameter, $g_{\mathrm{K} 1}$, by $50 \%$, in line with that observed experimentally in our laboratory (mRNA expression of KCNJ2/Kir2.1 reduced by $55 \%$ in fail $v s$ control myocytes, $p=0.01$, two-way ANOVA; $n=12$ [3]). Models were paced to steady-state at a frequency of $8 \mathrm{~Hz}$, followed by a quiescent period during which spontaneous activity could be observed. Changes in electrophysiological or $\mathrm{Ca}^{2+}$ handling behaviour were recorded. Models were coded in $\mathrm{C} / \mathrm{C}++$ and ran using the University of Leeds ARC3 High Performance Computing facilities.

\section{Results}

\subsection{Model Validation}

The developed cell model reproduces whole cell electrophysiology dynamics during control pacing at various cycle lengths and is stable over long simulation durations once steady-state is achieved. Action potential durations (APD, taken as the time to $90 \%$ repolarisation) at 1, 6 and $8 \mathrm{~Hz}$ were $42.49,52.29$ and $55.83 \mathrm{~ms}$ (Figure 3 ), and these values fell within expected experimental ranges. Representative simulated and experimental values for action potential and $\mathrm{Ca}^{2+}$ handling characteristics at $1 \mathrm{~Hz}$ are shown in Table 1.

Table 1. Simulated and experimental action potential and $\mathrm{Ca}^{2+}$ handling characteristics at $1 \mathrm{~Hz}$.

\begin{tabular}{ccc}
\hline \hline Parameter & Simulated & Experimental \\
\hline $\mathrm{APD}_{90}(\mathrm{~ms})$ & 42.49 & $46.3 \pm 8[8]$ \\
Resting potential $(\mathrm{mV})$ & -78.75 & $-76 \pm 2[8]$ \\
$\mathrm{AP}$ amplitude $(\mathrm{mV})$ & 114.48 & $108 \pm 7[9]$ \\
Systolic $\mathrm{Ca}^{2+}(\mathrm{uM})$ & 0.29 & $0.286 \pm 0.016[10]$ \\
$\mathrm{Ca}^{2+}$ transient duration $(\mathrm{ms})$ & 495 & $486[11]$ \\
\hline \hline
\end{tabular}

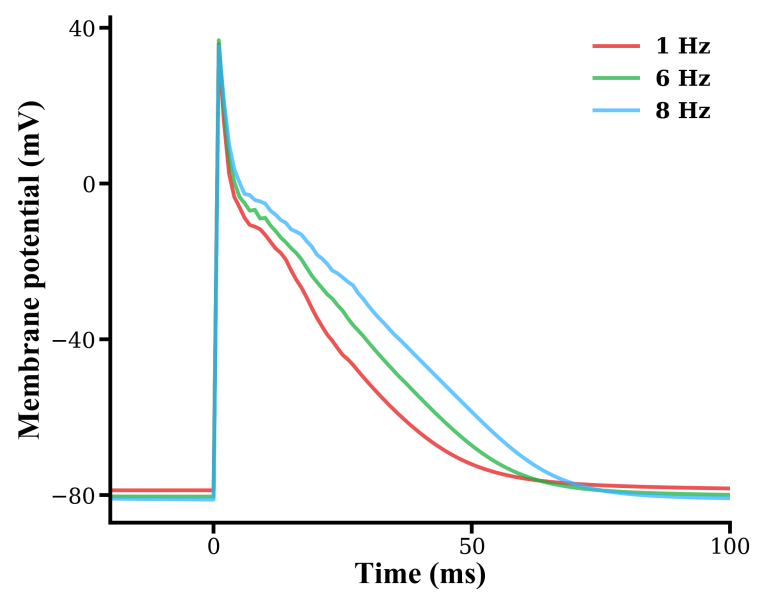

Figure 3. Simulated action potentials. The model reproduced action potential characteristics as observed experimentally at 1,6 and $8 \mathrm{~Hz}$. Average of 10 simulations shown per pacing frequency. 

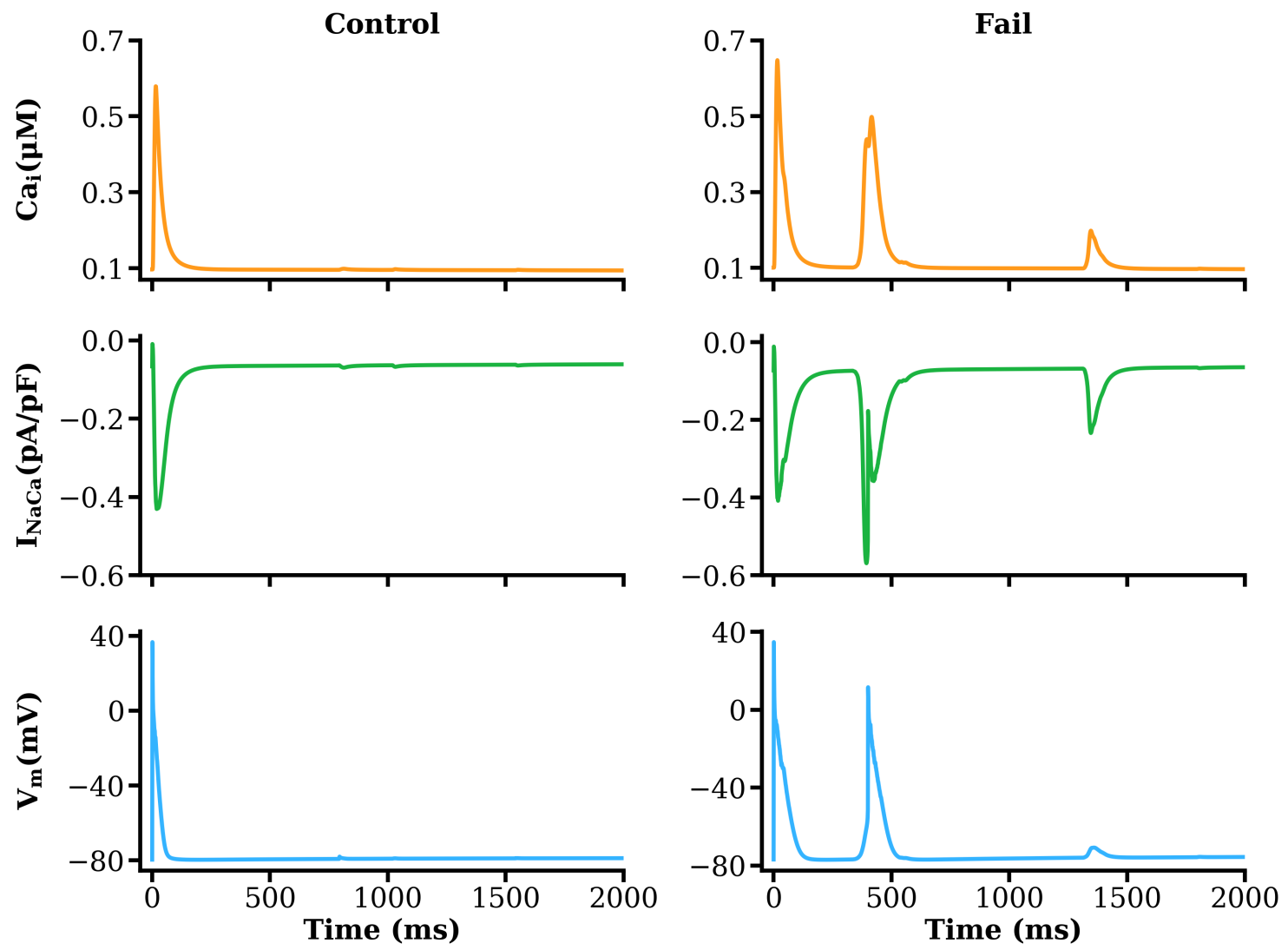

Figure 4. Effects of $I_{\mathrm{K} 1}$ downregulation on spontaneous activity. Left: Control simulations. No spontaneous activity occurs after steady-state pacing and so there is no diastolic depolarisation or erroneous $I_{\mathrm{NaCa}}$ activation. Right: Fail simulations. A large spontaneous $\mathrm{Ca}^{2+}$ release is seen, leading to inward $I_{\mathrm{NaCa}}$ and triggered activity at $400 \mathrm{~ms}$. Resting membrane potential is also depolarised.
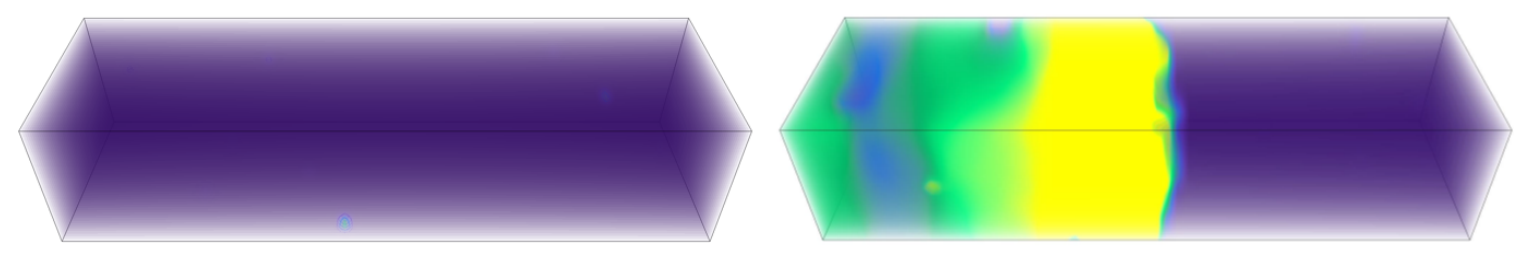

Figure 5. $\mathrm{Ca}^{2+}$ waves across the CRUs at $\mathrm{t}=500 \mathrm{~ms}$. Left: Control. No spontaneous $\mathrm{Ca}^{2+}$ wave is observed. Right: Fail. Spontaneous $\mathrm{Ca}^{2+}$ release from CRUs manifests as a propagating $\mathrm{Ca}^{2+}$ wave, resulting in triggered activity.

\subsection{Inward rectifier current downregula- tion promotes spontaneous $\mathrm{Ca}^{2+}$ re- lease}

Implementing a $50 \%$ reduction in $g_{\mathrm{K} 1}$, the $I_{\mathrm{K} 1}$ conductance parameter, resulted in a $57 \%$ prolongation of steadystate APD vs control, from 58.1 to $91.4 \mathrm{~ms}$. In addition, resting membrane potential in HF simulations was depo- larised by $3.3 \mathrm{mV}$, from -78.8 to $-75.5 \mathrm{mV}$.

Spontaneous activity is illustrated in Figure 4. In control simulations (left), there is no spontaneous $\mathrm{Ca}^{2+}$ release during the quiescent period after steady-state pacing (top), which is reflected by no erroneous activation of the sodium-calcium exchanger $\left(I_{\mathrm{NaCa}}\right.$, middle $)$ and no subsequent impact on membrane potential $\left(V_{\mathrm{m}}\right.$, bottom). Conversely, in HF simulations, a large spontaneous $\mathrm{Ca}^{2+}$ release $\left(\right.$ peak $\left.\left[\mathrm{Ca}^{2+}\right]_{\mathrm{i}}=0.498 \mathrm{uM}\right)$ activates forward-mode 
(depolarising) $I_{\mathrm{NaCa}}$ and results in a triggered action potential at $400 \mathrm{~ms}$. A second, smaller spontaneous release (peak $\left[\mathrm{Ca}^{2+}\right]_{\mathrm{i}}=0.198 \mathrm{uM}$ ) is observed later leading to a sub-threshold depolarisation of membrane potential by $8.19 \mathrm{mV}$ to $-70.82 \mathrm{mV}$. The manifestation of the larger release at $400 \mathrm{~ms}$ as a propagating $\mathrm{Ca}^{2+}$ wave is shown in Figure 5, as is its absence in the control simulation.

\section{Discussion and Conclusions}

We have developed a novel model of rat ventricular myocyte electrophysiology which has reproduced experimental variability in APD from other laboratories. The new model accounts for stochastic spatio-temporal $\mathrm{Ca}^{2+}$ handling dynamics alongside membrane ion channel electrophysiology and has provided insight into the mechanisms underlying increased spontaneous $\mathrm{Ca}^{2+}$ release in $\mathrm{HF}$ resulting from a reduced $I_{\mathrm{K} 1}$ current; that the resultant prolongation in APD allows more time for loading of the SR with $\mathrm{Ca}^{2+}$, promoting spontaneous $\mathrm{Ca}^{2+}$ release events. These releases result in forward-mode, depolarising sodium-calcium exchanger $\left(I_{\mathrm{NaCa}}\right)$ activity which in turn cause triggered action potentials. Combined with a destabilised membrane (resting membrane potential was depolarised in the HF simulation), this provides a trigger for arrhythmia development in failing myocytes.

Thus, the model provides a supplementary and standalone research tool which can be used to explore how sub-cellular changes associated with HF influence proarrhythmic activity at the single cell level. Incorporation into tissue-level simulation protocols may reveal the role of such remodelling in the development of ectopic activity and generation of arrhythmias at the organ level.

\section{Acknowledgements}

This research was funded by a University of Leeds PhD studentship, a Medical Research Council Strategic Skills Fellowship (MR/M014967/1) and a British Heart Foundation project grant (PG/16/74/32374).

\section{References}

[1] Saxon LA, Bristow MR, Boehmer J, Krueger S, Kass DA, De Marco T, Carson P, DiCarlo L, Feldman AM, Galle E, Ecklund F. Predictors of sudden cardiac death and appropriate shock in the comparison of medical therapy, pacing, and defibrillation in heart failure (companion) trial. Circulation 2006; 114:2766-2772.

[2] Roberts BN, Yang PC, Behrens SB, Moreno JD, Clancy CE. Computational approaches to understand cardiac electro- physiology and arrhythmias. American Journal of Physiology Heart and Circulatory Physiology 2012;303(7):H766H783.

[3] Benoist D, Stones R, Drinkhill M, Bernus O, White E. Arrhythmogenic substrate in hearts of rats with monocrotaline-induced pulmonary hypertension and right ventricular hypertrophy. American Journal of Physiology Heart and Circulatory Physiology 2011;300:H2230 H2237.

[4] Carnevali L, Sgoifo A. Vagal modulation of resting heart rate in rats: the role of stress, psychosocial factors, and physical exercise. Frontiers in Physiology 2014;5.

[5] Sadredini M, Danielsen TK, Aronsen JM, Manotheepan R, Hougen K, Sjaastad I, Stokke MK. Beta-adrenoceptor stimulation reveals ca2+ waves and sarcoplasmic reticulum ca2+ depletion in left ventricular cardiomyocytes from post-infarction rats with and without heart failure. Plos One 2016;11.

[6] Gattoni S, Roe AT, Frisk M, Louch WE, Niederer SA, Smith NP. The calcium-frequency response in the rat ventricular myocyte: an experimental and modelling study. Journal of Physiology London 2016;594:4193-4224.

[7] Colman MA, Pinali C, Trafford AW, Zhang HG, Kitmitto A. A computational model of spatio-temporal cardiac intracellular calcium handling with realistic structure and spatial flux distribution from sarcoplasmic reticulum and t-tubule reconstructions. Plos Computational Biology 2017;13.

[8] Perez-Cortes EJ, Islas AA, Arevalo JP, Mancilla C, Monjaraz E, Salinas-Stefanon EM. Modulation of the transient outward current (i-to) in rat cardiac myocytes and human $\mathrm{k}(\mathrm{v}) 4.3$ channels by mefloquine. Toxicology and Applied Pharmacology 2015;288:203-212.

[9] Ali RM, Al Kury LT, Yang KHS, Qureshi A, Rajesh M, Galadari S, Shuba YM, Howarth FC, Oz M. Effects of cannabidiol on contractions and calcium signaling in rat ventricular myocytes. Cell Calcium 2015;57:290-299.

[10] Lagadic-Gossmann D, Buckler KJ, LePrigent K, Feuvray D. Altered ca2+ handling in ventricular myocytes isolated from diabetic rats. American Journal of Physiology Heart and Circulatory Physiology 1996;270:H1529-H1537.

[11] Olson ML, Kargacin ME, Ward CA, Kargacin GJ. Effects of phloretin and phloridzin on ca2+ handling, the action potential, and ion currents in rat ventricular myocytes. Journal of Pharmacology and Experimental Therapeutics 2007; 321:921-929.

\section{Address for correspondence:}

Harley J. Stevenson-Cocks

School of Biomedical Sciences, Faculty of Biological Sciences, University of Leeds, Leeds, LS2 9JT, UK

bs12hjsc@leeds.ac.uk 